Check for updates

Cite this: J. Mater. Chem. C, 2018, 6, 3271

Received 4th January 2018,

Accepted 16th January 2018

DOI: $10.1039 / \mathrm{c} 8 \mathrm{tc} 00053 \mathrm{k}$

rsc.li/materials-c

\section{Cation, magnetic, and charge ordering in $\mathrm{MnFe}_{3} \mathrm{O}_{5}^{\dagger}$}

\author{
K. H. Hong, (D) a A. M. Arevalo-Lopez, ${ }^{b}$ M. Coduri, (D) ${ }^{c}$ G. M. McNally a and \\ J. P. Attfield (D)*a
}

\begin{abstract}
The recently-discovered high pressure material $\mathrm{MnFe}_{3} \mathrm{O}_{5}$ displays a rich variety of magnetically ordered states on cooling. Fe spins order antiferromagnetically below a Néel transition at $350 \mathrm{~K}$. A second transition at $150 \mathrm{~K}$ marks $\mathrm{Mn}$ spin order that leads to spin canting of some of the $\mathrm{Fe}$ spins and ferrimagnetism. A further transition at $60 \mathrm{~K}$ is driven by charge ordering of $\mathrm{Fe}^{2+}$ and $\mathrm{Fe}^{3+}$ over two inequivalent $\mathrm{Fe}$ sites, with further canting of all spins. Electrical resistivity measurements reveal semiconducting behaviour in $\mathrm{MnFe}_{3} \mathrm{O}_{5}$ with a change in activation energy at $285 \mathrm{~K}$.
\end{abstract}

\section{Introduction}

The variable $3 \mathrm{~d}^{n}$ configurations of Mn and Fe cations in spinel-type and other oxides give rise to many important applications as energy materials ${ }^{1}$ and in magnetism. Many manganese oxides are used as cathode materials in rechargeable batteries, e.g. $\mathrm{LiMn}_{2} \mathrm{O}_{4}$-spinel, $\mathrm{Li}_{2} \mathrm{MnO}_{3}$, and $\mathrm{NaMnO}_{2}$. The phosphate $\mathrm{LiFePO}_{4}$ has been commercialised as a lithium battery cathode and iron oxides such as $\mathrm{Fe}_{2} \mathrm{O}_{3}$ and magnetite, $\mathrm{Fe}_{3} \mathrm{O}_{4}$, have been explored as anode materials. ${ }^{2}$ Manganese and iron based spinels are also active catalysts for oxygen reduction/evolution reactions (ORR/OER) in fuel cells, metal-air batteries, and water-splitting cells. ${ }^{3}$

The spinel magnetite is also notable as the original magnetic material, and $\mathrm{Fe}_{3} \mathrm{O}_{4}$ has been studied intensively since Verwey's observation of a metal-insulator transition accompanied by a structural distortion. ${ }^{4}$ The low temperature structure has a complex charge and orbital ordering and weak Fe-Fe bonding interactions that form trimerons - linear orbital molecule clusters of three Fe ions. ${ }^{5,6}$ A related iron oxide, $\mathrm{Fe}_{4} \mathrm{O}_{5}$, was recently discovered using high temperature and high pressure synthesis, ${ }^{7}$ and has an incommensurate charge order at $150 \mathrm{~K}$, below which dimeron and trimeron-like groups of Fe ions are formed. ${ }^{8}$ Subsequent work has shown that $\mathrm{Fe}_{n} \mathrm{O}_{n+1}$ homologues with $n>4$ can also be made at pressure. ${ }^{9} \mathrm{M}^{2+} \mathrm{Fe}_{n-1} \mathrm{O}_{n+1}$ analogues of these materials with $\mathrm{M}^{2+}=\mathrm{Ca}$ were reported previously, ${ }^{10}$ and recent detailed studies of $\mathrm{CaFe}_{5} \mathrm{O}_{7}$ revealed

\footnotetext{
${ }^{a}$ Centre for Science at Extreme Conditions and School of Chemistry, University of Edinburgh, Mayfield Road, Edinburgh EH9 3JZ, UK. E-mail: j.p.attfield@ed.ac.uk

${ }^{b}$ Univ. Lille, CNRS, Centrale Lille, ENSCL, Univ. Artois, UMR 8181 - UCCS - Unité de Catalyse et Chimie du Solide, F-59000 Lille, France

${ }^{c}$ European Synchrotron Radiation Facility, 71 avenue des Martyrs, Grenoble, 38000 , France

$\dagger$ Electronic supplementary information (ESI) available: Tables of crystallographic results and powder synchrotron profile fit. See DOI: 10.1039/c8tc00053k
}

a coupled structural and magnetic transition at $360 \mathrm{~K}$ accompanied by $\mathrm{Fe}^{2+} / \mathrm{Fe}^{3+}$ charge ordering. ${ }^{11-13}$

We recently reported the synthesis at high pressures of the first $n>3 \mathrm{M}=\mathrm{Mn}$ material in this family, the $n=4$ member $\mathrm{MnFe}_{3} \mathrm{O}_{5} \cdot{ }^{14} \mathrm{MnFe}_{3} \mathrm{O}_{5}$ is isostructural with $\mathrm{Fe}_{4} \mathrm{O}_{5}$ and adopts the orthorhombic $\mathrm{Sr}_{2} \mathrm{Tl}_{2} \mathrm{O}_{5}$-type structure (space group $\mathrm{Cmcm}$ ) in which divalent cations occupy triangular prismatic sites within triangular channels in a network of corner and edge-sharing octahedra. $\mathrm{MnFe}_{3} \mathrm{O}_{5}$ showed two magnetic transitions - an antiferromagnetic transition at $350 \mathrm{~K}$ and a broad ferromagnetic transition at $150 \mathrm{~K}$ - indicating that complex spin-spin interactions are present. We report here a high resolution powder neutron diffraction study of $\mathrm{MnFe}_{3} \mathrm{O}_{5}$, revealing the $\mathrm{Mn} / \mathrm{Fe}$ chemical order and the temperature evolution of the magnetic behaviour between 5 and $400 \mathrm{~K}$, supported by low temperature powder synchrotron X-ray diffraction and the electrical resistivity measurements.

\section{Experimental}

$\mathrm{MnFe}_{3} \mathrm{O}_{5}$ was synthesised using high pressure and high temperature solid state synthesis. Powders of $\mathrm{MnO}$ and $\mathrm{Fe}_{3} \mathrm{O}_{4}$ were ground together in a $1: 1$ ratio, and were heated at $1400{ }^{\circ} \mathrm{C}$ in a Pt capsule for 20 min under $10 \mathrm{GPa}$ pressure, using a two-stage Walker-type module. Products were characterised by laboratory X-ray diffraction data collected with a Bruker D2 diffractometer using $\mathrm{Cu}-\mathrm{K}_{\alpha}$ radiation.

Magnetic measurements were carried out with a Quantum Design MPMS XL SQUID magnetometer. Magnetic susceptibility was recorded in zero field cooled (ZFC) and field cooled (FC) conditions between 2 and $400 \mathrm{~K}$ with an applied magnetic field of 5000 Oe. Hysteresis loops were measured at 2, 75, 300 and $400 \mathrm{~K}$. Electrical resistivity measurements were carried out with a Quantum Design PPMS, between 260 and $380 \mathrm{~K}$. 
High resolution time-of-flight neutron diffraction data were collected at the WISH beamline of the ISIS facility, with $50 \mathrm{mg}$ of powder from several high pressure runs packed into a vanadium can. Diffraction patterns were collected at 5, 75, 150, 300 and $400 \mathrm{~K}$ using a closed cycle refrigerator (CCR) with a hot stage. High resolution powder X-ray diffraction data were collected at the ID22 beamline of the ESRF with incident wavelength $0.39994 \AA$. A glass capillary with an outer diameter of $0.3 \mathrm{~mm}$ was used to contain the polycrystalline sample. Low temperature diffraction data were collected from 13 to $120 \mathrm{~K}$ using a liquid helium cryostat system.

\section{Results and discussion}

\section{Crystal and magnetic structures}

High resolution time-of-flight neutron diffraction experiments were carried out to determine the structure and magnetic behaviour of $\mathrm{MnFe}_{3} \mathrm{O}_{5}$. Crystal structure refinements show that the unit cell symmetry remains orthorhombic $\mathrm{Cmcm}$ at all temperatures. Occupancy refinements for the three cation sites, making use of the high neutron scattering contrast between Mn and Fe, showed that octahedral sites are occupied exclusively by $\mathrm{Fe}$, and the trigonal prism site is predominantly $\mathrm{Mn}$ with $6.3(4) \%$ substitution by Fe. Hence $\mathrm{MnFe}_{3} \mathrm{O}_{5}$ is $\mathrm{Mn} / \mathrm{Fe}$ ordered and the overall $\mathrm{Mn}_{0.94} \mathrm{Fe}_{3.06} \mathrm{O}_{5}$ composition of the present sample is close to the ideal stoichiometry.

Magnetisation measurements (Fig. 1) show a maximum at $350 \mathrm{~K}$ and a broad magnetic upturn signifying a ferro- or ferri-magnetic ordering at $150 \mathrm{~K}$ as reported previously. ${ }^{14}$ This is corroborated by the saturated magnetisations observed in hysteresis loops at 2 and $75 \mathrm{~K}$.

The neutron diffraction patterns shown in Fig. 2 reveal the appearance of magnetic reflections when cooled below $400 \mathrm{~K}$. The magnetic structures in the different regimes were solved and Rietveld fitted to the neutron diffraction patterns, as shown for the $5 \mathrm{~K}$ pattern in Fig. 3. The magnetic reflections from all the magnetic phases of $\mathrm{MnFe}_{3} \mathrm{O}_{5}$ were indexed with a

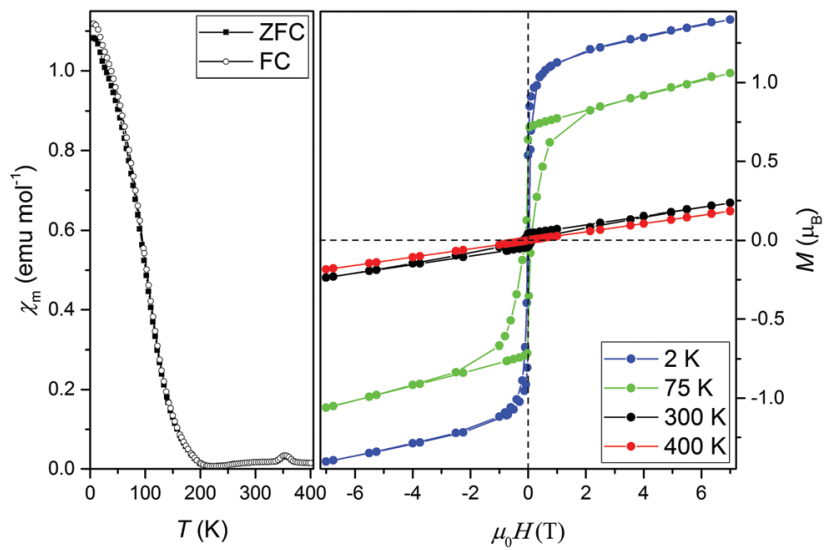

Fig. 1 (left) Zero-field cooled and field cooled magnetisation measurements between 2 and $400 \mathrm{~K}$. (right) Magnetisation-field hysteresis loops for $\mathrm{MnFe}_{3} \mathrm{O}_{5}$ measured at 2, 75, 300 and $400 \mathrm{~K}$.

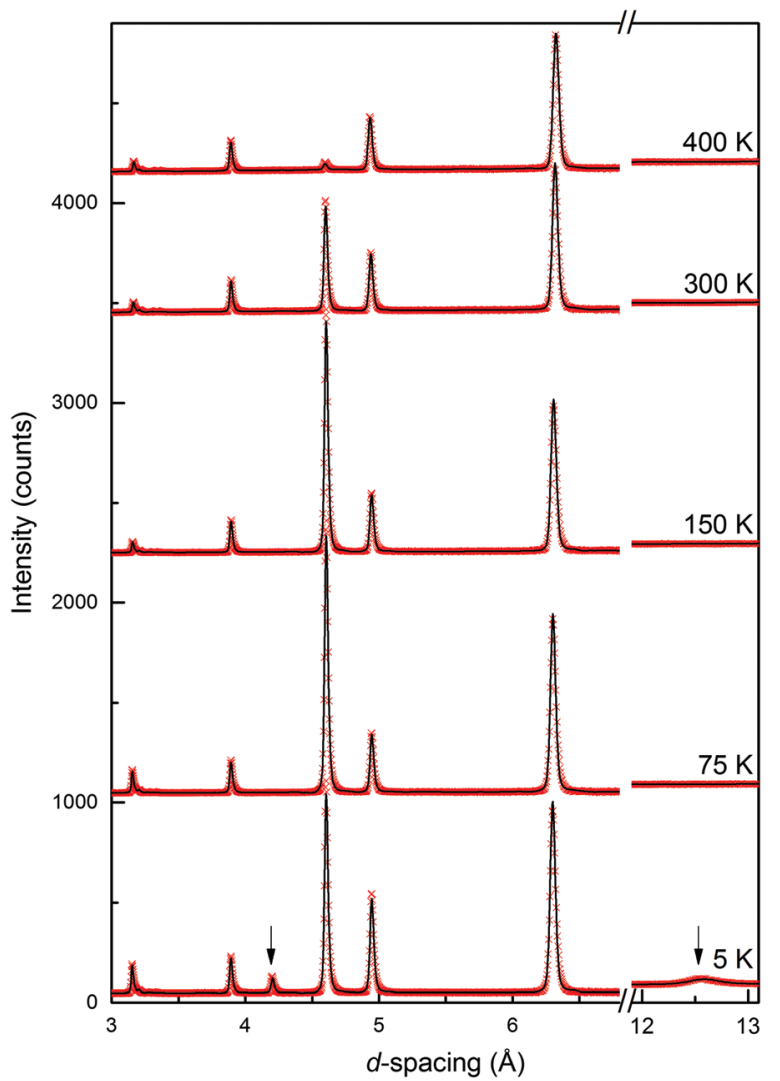

Fig. 2 Temperature evolution of the neutron diffraction pattern of $\mathrm{MnFe}_{3} \mathrm{O}_{5}$. Magnetic contributions appear at $300 \mathrm{~K}$, and arrows indicate additional magnetic peaks at $5 \mathrm{~K}$, with $h k l=(001)$ and (003) at $d$-spacing $=$ 12.6 and $4.2 \AA$, respectively.

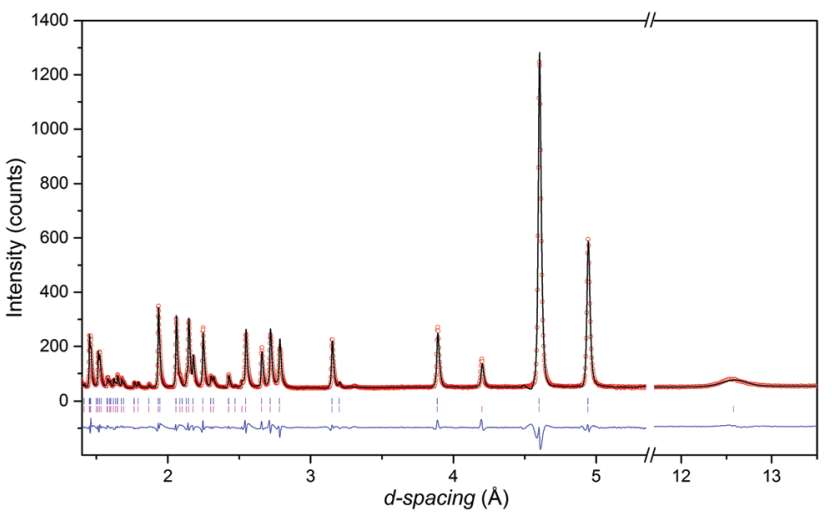

Fig. 3 Rietveld refinement of high resolution neutron diffraction patterns of $\mathrm{MnFe}_{3} \mathrm{O}_{5}$ obtained at $5 \mathrm{~K}$, with upper tick marks indicating nuclear peaks and lower presenting magnetic reflections. $\left(R_{\mathrm{wp}}=12.9 \%\right.$ and $R_{\mathrm{p}}=12.6 \%$ ).

propagation vector of (000), and the structures obtained at 5 , 75 and $300 \mathrm{~K}$ are presented in Fig. 4a. At 150 and $300 \mathrm{~K}$, the spins at the two independent $\mathrm{Fe}$ sites in $\mathrm{MnFe}_{3} \mathrm{O}_{5}$ are both found to be ordered antiferromagnetically parallel to the $c$-axis, whilst the Mn spins remain disordered. This confirms that a Néel transition is observed at $T_{\mathrm{N}}=350 \mathrm{~K}$ in the magnetisation measurements (Fig. 1). 
a)

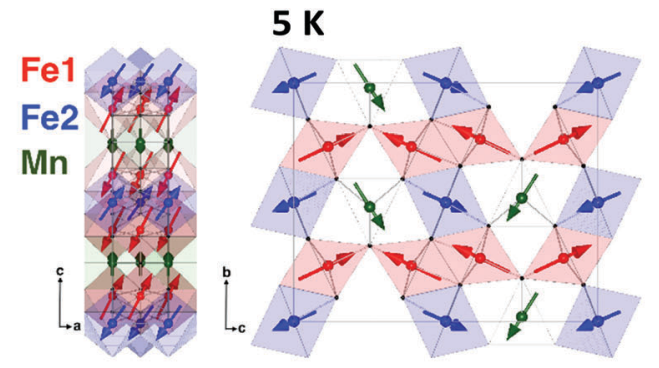

$75 \mathrm{~K}$

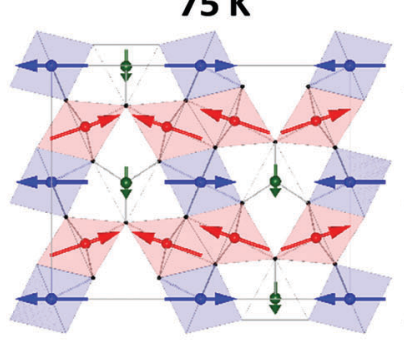

b)

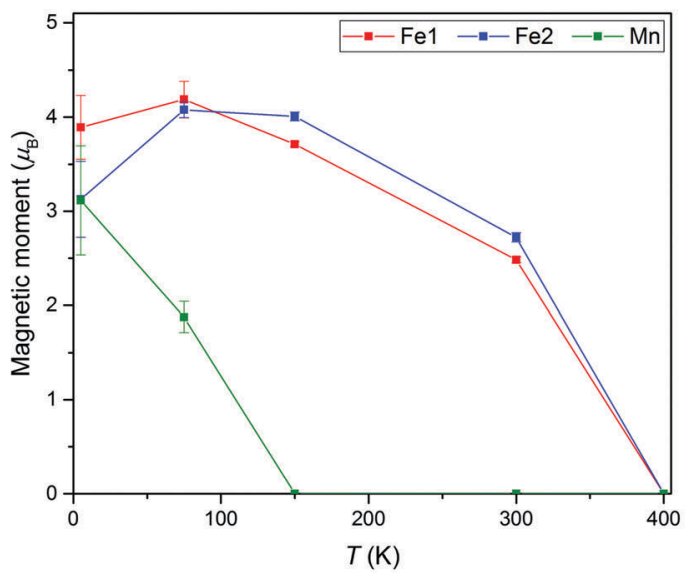

c)

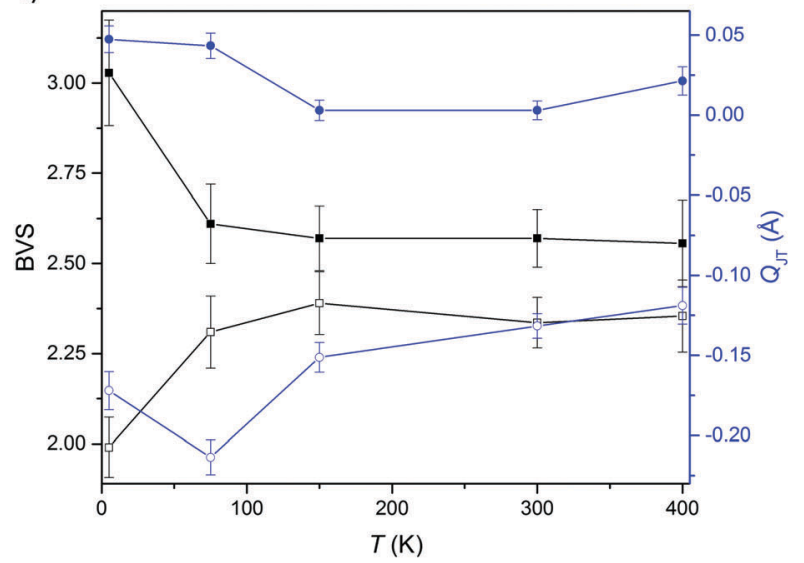

Fig. 4 (a) Magnetic structures of $\mathrm{MnFe}_{3} \mathrm{O}_{5}$ at 5, 75 and $300 \mathrm{~K}$. The network of $\mathrm{FeO}_{6}$ octahedra is shown, with $\mathrm{Mn}^{2+}$ in trigonal prismatic sites within channels parallel to the $a$-axis. (b) The temperature evolution of the ordered $\mathrm{Mn}, \mathrm{Fe} 1$ and $\mathrm{Fe} 2$ magnetic moments. (c) Temperature evolution of BVS and $Q_{J T}$, with closed/open symbols representing Fe1/Fe2 sites.

Fits to the $75 \mathrm{~K}$ diffraction data showed additional spin ordering of the Mn site, with moments aligned ferromagnetically along the $b$ axis below $T_{\mathrm{Mn}}=150 \mathrm{~K}$. The order of the Mn site moment leads the spins of the nearest Fe site - Fe1 to cant towards the $b$ axis. The $b$-components of Fe1 spins are antiparallel to those of $\mathrm{Mn}$, resulting in a net magnetisation of $\sim 0.5 \mu_{\mathrm{B}}$ per $\mathrm{MnFe}_{3} \mathrm{O}_{5}$ formula unit, which is consistent with the increase in magnetisation on cooling from 300 to $75 \mathrm{~K}$ shown in Fig. 1. A similar spin canting was reported in $\mathrm{Fe}_{4} \mathrm{O}_{5}{ }^{8}$

The additional magnetic reflections observed in the $5 \mathrm{~K}$ neutron diffraction patterns reveal another change in the magnetic structure in $\mathrm{MnFe}_{3} \mathrm{O}_{5}$. The onset for this third magnetic transition appears to be the divergence between zero-field cooled and field cooled susceptibilities at $\sim 60 \mathrm{~K}$ in the susceptibility data (Fig. 1). An increase in the ordered Mn moment leads to further canting of all the spins, with both of the Fe sites canted antiferromagnetically towards the $a$ axis and ferromagnetically along $b$. In addition, the Mn spins become canted antiferromagnetically to the $c$ axis. The magnetic components of the $\mathrm{Mn}$ and the $\mathrm{Fe} 2$ sites on the $b$ axis are antiparallel to Fe1, as shown in Fig. 4a. This enhances the magnetisation along the $b$ axis to $\sim 0.6 \mu_{\mathrm{B}}$ per $\mathrm{MnFe}_{3} \mathrm{O}_{5}$ formula unit. The thermal evolution of the magnitude of the ordered moments in $\mathrm{MnFe}_{3} \mathrm{O}_{5}$ are shown in Fig. $4 \mathrm{~b}$, and the values of the ordered components and other refinement results are given in ESI. $\dagger$

The $300 \mathrm{~K}$ magnetic structure reveals dominant antiferromagnetic Fe1-Fe2 interactions; from direct exchange via overlap of half-filled $\mathrm{t}_{2 \mathrm{~g}}$ orbitals through edge-sharing of $\mathrm{FeO}_{6}$ octahedra, and through superexchange mediated by $\mathrm{Fe}-\mathrm{O}-\mathrm{Fe}$ connections at shared corners. Each $\mathrm{Mn}$ spin is coupled to $4 \mathrm{Fe} 1$ and $4 \mathrm{Fe} 2$ spins via $\mathrm{Mn}-\mathrm{O}-\mathrm{Fe}$ bridges, and frustration of both of these interactions leads to an almost perpendicular alignment of the Mn moments at $75 \mathrm{~K}$, although Fe1 spin canting occurs such that their components in the $b$-direction are antiferromagnetically coupled to the Mn spins. Further canting occurs at $5 \mathrm{~K}$ as the Mn spins become more fully ordered and $\mathrm{Fe} 1$ and $\mathrm{Fe} 2$ spins cant out of the $b c$-plane. This magnetic order breaks the mirror-plane symmetries of the lattice perpendicular to the $a$ and $c$ axes, so an exchangestrictive distortion might be expected but is not observed within the resolution of the present data.

The possibility of internal electronic distortions was investigated by using the Bond Valence Sum (BVS) method to estimate oxidation states for the Fe sites via a standard interpolation method $^{15,16}$ with bond distances derived from the neutron refinements. The BVS results in Fig. 4c show that both Fe1 and $\mathrm{Fe} 2$ sites have mixed $\mathrm{Fe}^{2+} / \mathrm{Fe}^{3+}$ charge states at $75-400 \mathrm{~K}$, but charge ordering is evident at $5 \mathrm{~K}$ with $\mathrm{Fe} 1$ and $\mathrm{Fe} 2$ respectively tending to $\mathrm{Fe}^{3+}$ and $\mathrm{Fe}^{2+}$ states. The effect of Jahn Teller distortion $\left(Q_{\mathrm{JT}}\right)$ is also calculated for both Fe sites. Charge localisation as $\mathrm{Fe}^{2+}$ is expected to lead to Jahn Teller compression of the $\mathrm{Fe}_{2} \mathrm{O}_{6}$ octahedron (negative values of the $Q_{\mathrm{JT}}$ parameter reported in ref. 5), and although no large changes are observed on cooling below $75 \mathrm{~K}$, the negative $Q_{\mathrm{JT}}$ for $\mathrm{Fe} 2\left(\mathrm{Fe}^{2+}\right)$ and near zero value for $\mathrm{Fe} 1\left(\mathrm{Fe}^{3+}\right)$ corroborate the BVS charge ordering results. Hence a $\mathrm{Fe}^{2+} / \mathrm{Fe}^{3+}$ charge ordering transition occurs at $T_{\mathrm{CO}}=60 \mathrm{~K}$ in $\mathrm{MnFe}_{3} \mathrm{O}_{5}$, with concomitant spin canting. As the $\mathrm{Fe}^{2+}$ and $\mathrm{Fe}^{3+}$ states respectively localise at inequivalent $\mathrm{Fe} 2$ and $\mathrm{Fe} 1$ sites there 


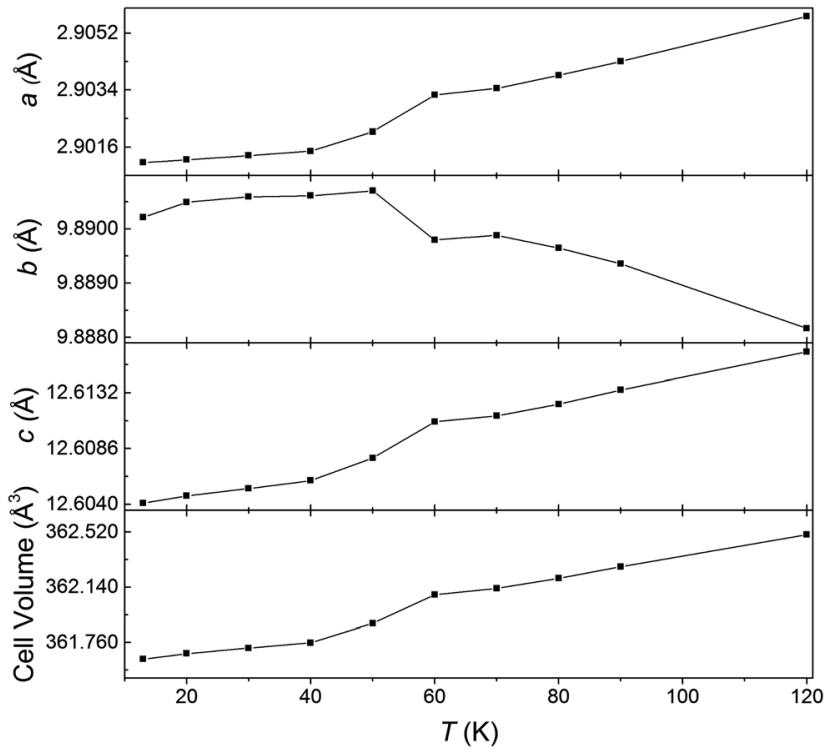

Fig. 5 Changes in the lattice parameters and cell volume obtained from powder synchrotron $\mathrm{X}$-ray diffraction experiments.

is no symmetry-breaking distortion associated with the charge order, or with the $\mathrm{Fe}^{2+}$ orbital order, although the associated $5 \mathrm{~K}$ spin order does break the $\mathrm{Cmcm}$ lattice symmetry as noted above.

Our previous study of the crystal structure using powder synchrotron X-ray diffraction data showed that anisotropic thermal expansion of the lattice parameters is observed over the temperature range 90-400 K. Further data collected between 15 and $120 \mathrm{~K}$ here confirm that the orthorhombic $\mathrm{Cmcm}$ structure persists to low temperatures. A discontinuity in the refined lattice parameters and cell volume is observed at $60 \mathrm{~K}$, (Fig. 5) corresponding to the divergence in the ZFC and FC magnetisation measurements. This likely marks the onset of the charge ordering observed in the $5 \mathrm{~K}$ but not the $75 \mathrm{~K}$ neutron diffraction data.

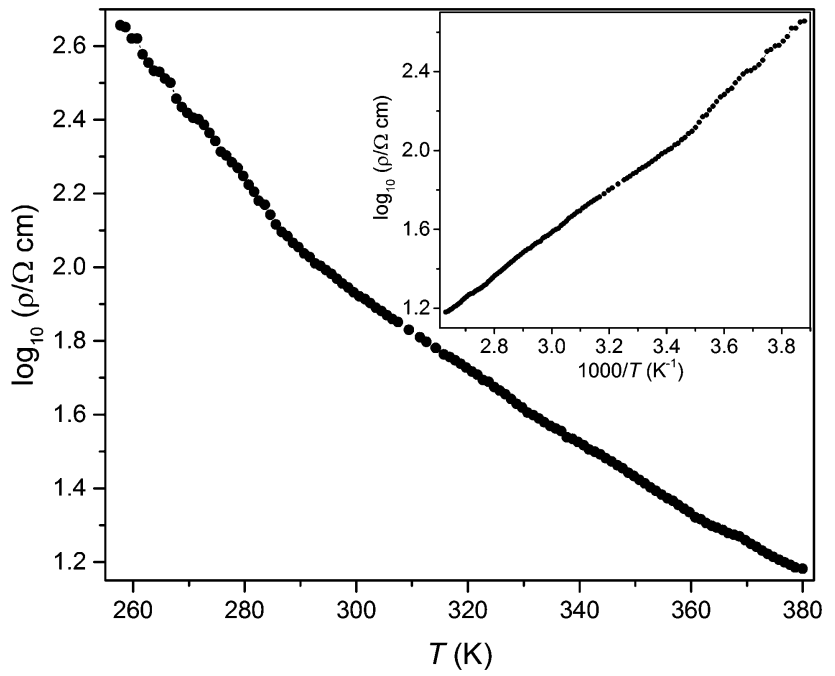

Fig. 6 Log of electrical resistivity of $\mathrm{MnFe}_{3} \mathrm{O}_{5}$ measured between 260 and $380 \mathrm{~K}$, with insert showing the plot against reciprocal temperature.

\section{Electrical properties}

Electrical resistivity measurement of a polycrystalline pellet of $\mathrm{MnFe}_{3} \mathrm{O}_{5}$ shows semiconducting behaviour, with the resistivity increasing when cooled (Fig. 6). The sample resistance was too great to be measured below $260 \mathrm{~K}$. Two linear regions are observed in the inset plot of $\log$ (resistivity) vs. inverse temperature, with a change of slope near $285 \mathrm{~K}$. Fitting the Arrhenius equation $\rho=A \exp \left(E_{\mathrm{a}} / k_{\mathrm{B}} T\right)$ to the two linear regions gives the activation energy $E_{\mathrm{a}}$ as $210 \mathrm{meV}$ above and $280 \mathrm{meV}$ below the $285 \mathrm{~K}$ crossover. This temperature does not match any of the observed magnetic transition temperatures, and may correspond to a change from defect-dominated to intrinsic bandgap conduction.

The presence of structural channels within a framework of redox-active $\mathrm{FeO}_{6}$ octahedra, and moderate electrical conductivity, suggests that $\mathrm{MnFe}_{3} \mathrm{O}_{5}$ would be worth investigating as a battery electrode material. Full reduction of $\mathrm{Fe}^{3+}$ to $\mathrm{Fe}^{2+}$ through lithium insertion would give $\mathrm{Li}_{2} \mathrm{MnFe}_{3} \mathrm{O}_{5}$, and as cycling between this and the parent phase does not involve oxidation of $\mathrm{Mn}^{2+}$ then structural degradation from formation of Jahn-Teller active $\mathrm{Mn}^{3+}$ is avoided. However, alternative methods to high pressure synthesis are likely to be needed to make sufficient quantities of $\mathrm{MnFe}_{3} \mathrm{O}_{5}$ for practical battery research.

\section{Conclusions}

$\mathrm{MnFe}_{3} \mathrm{O}_{5}$ is found to have a rich magnetic ordered behaviour between 5 and $400 \mathrm{~K}$. On cooling from high temperatures, the moments at the iron sites order with an antiferromagnetic arrangement at $T_{\mathrm{N}}=350 \mathrm{~K}$, but additional order of $\mathrm{Mn}$ moments below $T_{\mathrm{Mn}}=150 \mathrm{~K}$ leads to canting of Fe1 spins and a significant ferrimagnetic moment. A similar canting of $\mathrm{Fe}$ (and Re) spins due to low temperature order of $\mathrm{Mn}^{2+}$ moments was reported in the double perovskite $\mathrm{Mn}_{2} \mathrm{FeReO}_{6} \cdot{ }^{17} \mathrm{Fe}^{2+} / \mathrm{Fe}^{3+}$ charge ordering drives a further transition at $T_{\mathrm{CO}}=60 \mathrm{~K}$ with additional canting of all spins. Similar charge and orbital orders are observed in $\mathrm{Fe}_{3} \mathrm{O}_{4}$ and $\mathrm{Fe}_{4} \mathrm{O}_{5}$. Electrical resistivity measurements reveal semiconducting behaviour in $\mathrm{MnFe}_{3} \mathrm{O}_{5}$ and a small change in the activation energy at $285 \mathrm{~K}$ may correspond to a crossover from defect to intrinsic conduction regimes. $\mathrm{MnFe}_{3} \mathrm{O}_{5}$ is worth further investigation as a potential lithium battery electrode material.

\section{Conflicts of interest}

There are no conflicts to declare.

\section{Acknowledgements}

We acknowledge financial support from European Research Council (ERC), Engineering and Physical Sciences Research Council (EPSRC) and Science, Technology Facilities Council (STFC) and the ESRF for provision of beamtime. We would also like to thank Andy Fitch (ESRF), Pascal Manuel (ISIS) and 
James Cumby, Alexander J. Browne, Giuditta Perversi and Paul M. Sarte (Edinburgh) for assistance provided.

\section{References}

1 N. Yabuuch and S. Komaba, Sci. Technol. Adv. Mater., 2014, 15, 043501.

2 L. Zhang, H. B. Wu and X. W. Lou, Adv. Energy Mater., 2013, 4, 1300958.

3 Q. Zhao, Z. Yan, C. Chen and J. Chen, Chem. Rev., 2017, 117, 10121-10211.

4 E. J. W. Verwey, Nature, 1939, 144, 327-328.

5 M. S. Senn, J. P. Wright and J. P. Attfield, Nature, 2012, 481, 173-176.

6 G. Perversi, J. Cumby, E. Pachoud, J. P. Wright and J. P. Attfield, Chem. Commun., 2016, 52, 4864.

7 B. Lavina, P. Dera, E. Kim, Y. Meng, R. T. Downs, P. F. Weck, S. R. Sutton and Y. Zhao, Proc. Natl. Acad. Sci. U. S. A., 2011, 108, 17281-17285.

8 S. V. Ovsyannikov, M. Bykov, E. Bykova, D. P. Kozlenko, A. A. Tsirlin, A. E. Karkin, V. V. Shchennikov, S. E. Kichanov,
H. Gou, A. M. Abakumov, R. Egoavil, J. Verbeeck, C. McCammon, V. Dyadkin, D. Chernyshov, S. van Smaalen and L. S. Dubrovinsky, Nat. Chem., 2016, 1-8.

9 B. Lavina and Y. Meng, Sci. Adv., 2015, 1, e1400260.

10 O. Evrard, B. Malaman and F. Jeannot, J. Solid State Chem., 1980, 35, 112-119.

11 C. Delacotte, F. Hüe, Y. Bréard, S. Hébert, O. Pérez, V. Caignaert, J. M. Greneche and D. Pelloquin, Inorg. Chem., 2014, 53, 10171-10177.

12 C. Delacotte, Y. Bréard, V. Caignaert, V. Hardy, J. M. Greneche, S. Hébert, E. Suard and D. Pelloquin, Key Eng. Mater., 2014, 617, 237-240.

13 C. Delacotte, Y. Bréard, V. Caignaert, V. Hardy, J. M. Greneche, S. Hébert, E. Suard and D. Pelloquin, J. Solid State Chem., 2017, 247, 13-19.

14 K. H. Hong, G. M. McNally, M. Coduri and J. P. Attfield, Z. Anorg. Allg. Chem., 2016, 642, 1355-1358.

15 J. P. Attfield, Solid State Sci., 2006, 8, 861-867.

16 I. D. Brown, J. Appl. Crystallogr., 1996, 29, 479-480.

17 A. M. Arévalo-López, G. M. McNally and J. P. Attfield, Angew. Chem., Int. Ed., 2015, 54, 12074-12077. 